\section{Commentary: Is there a surgeon in the house?}

\author{
Edward Y. Sako, MD, PhD
}

Ram and colleagues ${ }^{1}$ present an interesting and timely study. They examine outcomes of patients initially treated at cardiac centers with on-site cardiac surgery versus those without. Strengths of the study include multicenter data collection using a registry that is country-wide among public hospitals, and medium-term follow-up at least for mortality using their national population register.

It is important to note that the study population consisted of patients with multivessel disease (specifically defined) who underwent some form of revascularization. Patients undergoing medical therapy were excluded. The authors found that in the centers with on-site cardiac surgery, more than one half of the patients were referred for coronary artery bypass surgery. In those without, only 35\% were referred for surgery. The unadjusted 6-year survival was greater in patients treated at centers with on-site cardiac surgery and was driven in part by the larger number of patients who underwent surgery. In addition, the authors found that among patients referred for surgery, there was a greater mortality risk for those coming from the nonsurgical centers. Possible explanations included delays in undergoing surgery and referral of only the more complex, and therefore, greater-risk patients.

Much was made in the discussion of the influence and possible benefit of a heart-team approach. A heart-team approach implies that a surgeon is actively involved in the decision-making process before any form of revascularization. It is not clear that this took place in the centers with onsite surgery, particularly considering the era in which the patients were studied. More likely, the difference lies in the convenience and accessibility of surgery in the on-site centers compared with the increased effort and delay in

\footnotetext{
From the Department of Cardiothoracic Surgery, University of Texas Health Science Center at San Antonio, San Antonio, Tex.

Disclosures: Dr Sako has a financial relationship with Medtronic, Inc.

The Journal policy requires editors and reviewers to disclose conflicts of interest and to decline handling or reviewing manuscripts for which they may have a conflict of interest. The editors and reviewers of this article have no conflicts of interest.

Received for publication May 12, 2020; revisions received May 12, 2020; accepted for publication May 12, 2020; available ahead of print May 26, 2020.

Address for reprints: Edward Y. Sako, MD, PhD, Department of Cardiothoracic Surgery, University of Texas Health Science Center at San Antonio, Mail Code 7841, 7703 Floyd Curl Dr, San Antonio, TX 78229-3900 (E-mail: sako@uthscsa.edu). J Thorac Cardiovasc Surg 2022;163:1863

$0022-5223 / \$ 36.00$

Copyright (C) 2020 by The American Association for Thoracic Surgery

https://doi.org/10.1016/j.jtcvs.2020.05.042
}

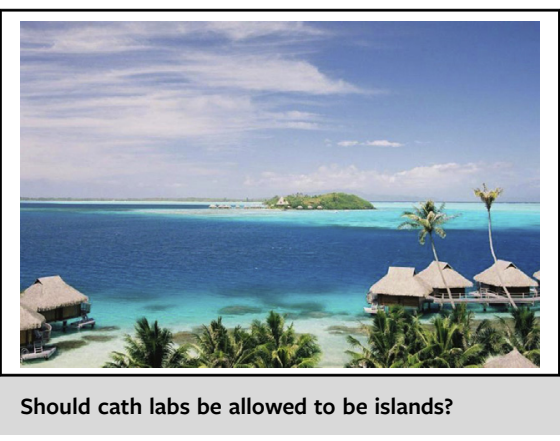

CENTRAL MESSAGE

Beware the standalone cath lab.

getting patients from the centers without on-site surgery to a surgical center. This is acknowledged in the limitations section. However, it is almost a given that surgeon input did not take place in centers without on-site surgery.

Also missing from the analysis is the larger picture of the nature and location of the centers relative to one another. For example, are there centers without on-site surgery that do not have the volume to support a reasonable surgical program? Yet, do those centers, perhaps in rural areas, still play a valuable role in providing some level of immediate cardiac care? Excluded from the group were patients treated with "primary percutaneous coronary intervention." This represented a group of patients with acute coronary syndromes or evolving ischemia that may have benefited from urgent percutaneous coronary intervention. Beyond door to balloon, is there an advantage to reducing onset of pain to balloon?

Nonetheless, this intriguing study should prompt the examination of such outcomes in other regions. It confirms the biased perception that heart centers without on-site cardiac surgery will tend to do more catheter-based therapy in lieu of surgery and that this may have medium- to long-term consequences.

\section{Reference}

1. Ram E, Raanani E, Klempfner R, Peled Y, Sternik L, Segev A. Midterm outcomes of patients with multivessel disease treated at centers with and without on-site cardiac surgery services. J Thorac Cardiovasc Surg. 2022;163:1852-61.e3. 